This is an electronic reprint of the original article. This reprint may differ from the original in pagination and typographic detail.

\author{
Author(s): Badran, Hussam; Scholey, Catherine; Auranen, Kalle; Grahn, Tuomas; Greenlees, Paul; \\ Herzan, Andrej; Jakobsson, Ulrika; Julin, Rauno; Juutinen, Sakari; Konki, Joonas; Leino, \\ Matti; Mallaburn, Michael; Pakarinen, Janne; Papadakis, Philippos; Partanen, Jari; \\ Peura, Pauli; Rahkila, Panu; Sandzelius, Mikael; Sarén, Jan; Sorri, Juha; Stolze, Sanna; \\ Uusitalo, Juha
}

Title: $\quad$ Confirmation of the new isotope $178 \mathrm{~Pb}$

Year: $\quad 2016$

Version:

Please cite the original version:

Badran, H., Scholey, C., Auranen, K., Grahn, T., Greenlees, P., Herzan, A., Jakobsson, U., Julin, R., Juutinen, S., Konki, J., Leino, M., Mallaburn, M., Pakarinen, J., Papadakis, P., Partanen, J., Peura, P., Rahkila, P., Sandzelius, M., Sarén, J., . . Uusitalo, J. (2016). Confirmation of the new isotope 178Pb. Physical Review C, 94(5), Article 054301. https://doi.org/10.1103/PhysRevC.94.054301

All material supplied via JYX is protected by copyright and other intellectual property rights, and duplication or sale of all or part of any of the repository collections is not permitted, except that material may be duplicated by you for your research use or educational purposes in electronic or print form. You must obtain permission for any other use. Electronic or print copies may not be offered, whether for sale or otherwise to anyone who is not an authorised user. 


\title{
Confirmation of the new isotope ${ }^{178} \mathrm{~Pb}$
}

\author{
H. Badran, ${ }^{1, *}$ C. Scholey, ${ }^{1}$ K. Auranen, ${ }^{1, \dagger}$ T. Grahn, ${ }^{1}$ P. T. Greenlees, ${ }^{1}$ A. Herzan,,${ }^{1,}$ U. Jakobsson,,${ }^{2,}$ R. Julin, ${ }^{1}$ S. Juutinen, ${ }^{1}$ \\ J. Konki, ${ }^{1}$ M. Leino, ${ }^{1}$ M. Mallaburn,,${ }^{1,3}$ J. Pakarinen, ${ }^{1}$ P. Papadakis, ${ }^{1}$ J. Partanen, ${ }^{1}$ P. Peura,,${ }^{1} \|$ P. Rahkila, ${ }^{1}$ M. Sandzelius, ${ }^{1}$ \\ J. Sarén, ${ }^{1}$ J. Sorri, ${ }^{1}$ S. Stolze, ${ }^{1}$ and J. Uusitalo ${ }^{1}$ \\ ${ }^{1}$ University of Jyväskylä, Department of Physics, P.O. Box 35, FI-40014, Jyväskylä, Finland \\ ${ }^{2}$ Department of Physics, KTH-Royal Institute of Technology, SE-10691 Stockholm, Sweden \\ ${ }^{3}$ University of Manchester, Manchester M13 9PL, United Kingdom \\ (Received 9 June 2016; revised manuscript received 14 September 2016; published 2 November 2016)
}

\begin{abstract}
The extremely neutron-deficient isotope ${ }^{178} \mathrm{~Pb}$ has been produced. The GREAT spectrometer at the focal plane position of the gas-filled separator RITU was used to study the $\alpha$ decay of ${ }^{178} \mathrm{~Pb}$ and its $\alpha$-decay chain through $\alpha-\alpha$ correlations. The $\alpha$ decay was measured to have an energy and half-life of $E_{\alpha}=7610(30) \mathrm{keV}$ and $t_{1 / 2}=$ $0.21_{-0.08}^{+0.21} \mathrm{~ms}$, respectively. The half-life is consistent with recent theoretical calculations using the Coulomb and proximity potential model. The $\alpha$-decay reduced width and hindrance factor for ${ }^{178} \mathrm{~Pb}$ were deduced and correspond to an unhindered $\Delta l=0$ transition. In addition, the mass excess of ${ }^{178} \mathrm{~Pb}$ and the $\alpha$-decay $Q$ value were calculated from the experimental results and compared to theoretical values.
\end{abstract}

DOI: 10.1103/PhysRevC.94.054301

\section{INTRODUCTION}

While the search for the next proton closed shell continues, $\mathrm{Pb}$ at $Z=82$ is the heaviest well-established proton shell closure which is experimentally accessible. Information gained from studying the $\alpha$ decay of $\mathrm{Pb}$ isotopes can test the robustness of this proton shell closure over a wide variation of neutron numbers. The most neutron-deficient lead isotope is ${ }^{178} \mathrm{~Pb}$ [1], located 30 mass units away from the stable doubly magic nucleus ${ }^{208} \mathrm{~Pb}$. On the other hand, the most neutron-rich $\mathrm{Pb}$ nucleus for which decay data have been collected is ${ }^{216} \mathrm{~Pb}$ [2], which is a mere 8 mass units from the doubly magic core. These facts show that producing $\mathrm{Pb}$ nuclei with a neutron excess is experimentally difficult, while $\mathrm{Pb}$ nuclei with a neutron deficit are more accessible, though still experimentally challenging. Below $N=113$ lead nuclei have $\alpha$-decay branches from their ground states, which have been measured and hence allow ground-state properties to be studied despite relatively low production yields.

Hindrance factors, $\alpha$-decay reduced widths, and partial half-lives reveal structural changes between the parent and child nuclei. Systematic studies of $\alpha$-decay reduced widths of even-even lead nuclei and also of radon, polonium, mercury, and platinum nuclei, which are two and four protons away from the closed shell, have been discussed previously in Refs. [3-6]. The general finding for $\mathrm{Pb}$ nuclei is that the $\alpha$-decay reduced widths are smaller than those of $\mathrm{Rn}, \mathrm{Po}, \mathrm{Hg}$, and $\mathrm{Pt}$ neighbors and that deviations from this trend are brought about by the

\footnotetext{
*hussam.h.badran@jyu.fi

${ }^{\dagger}$ Present address: Argonne National Laboratory, 9700 Cass Ave, Lemont, IL 60439, USA.

${ }^{\ddagger}$ Present address: Oliver Lodge Laboratory, University of Liverpool, Liverpool L69 7ZE, UK.

\$Present address: Laboratory of Radiochemistry, Department of Chemistry, P.O. Box 55, FI-00014 Univeristy of Helsinki, Finland.

"Present address: Helsinki Institute of Physics, University of Helsinki, P.O. Box 64, 00014 Helsinki, Finland.
}

presence of intruder states and configuration mixing, due to shape coexistence. Andreyev et al. [6] discussed the $\alpha$-particle preformation probability in relation to the neutron pairing energy for the nuclei in this region, which demonstrated the effect of the shell closures of $N=126$ and $Z=82$ on these values. As reported in Ref. [6], it is both the proton and neutron pairing gaps that effect the $\alpha$-particle preformation probability; hence it would be interesting to extend such a study beyond the proton dripline to observe the effect of weak proton binding on the $\alpha$-particle preformation probability, when approaching $N=82$. Recently the validity of the Geiger-Nuttall rule has also been brought into question by Qi et al. [7]. The importance of identifying the microscopic basis of the Geiger-Nuttall (GN) coefficients and the need for more systematic data points with more accurate $\alpha$-decay energies and half-lives is evident.

This paper reports on the confirmation of the new isotope ${ }^{178} \mathrm{~Pb}$, evidence for which was originally presented by Batchelder et al. [1], advancing the $\mathrm{Pb}$ systematics to further neutron deficiency. The $\alpha$-decay energy and halflife are presented and put in a systematic context and are also compared to recent theoretical calculations [8]. Two events were previously assigned to the $\alpha$ decay of ${ }^{178} \mathrm{~Pb}$ in Ref. [1], but only one of these events was correlated to a known daughter $\alpha$-decay event. The present work confirms and extends these preliminary findings with improved accuracy.

\section{EXPERIMENTAL DETAILS}

The lead nuclei of interest were produced using the fusionevaporation reaction ${ }^{104} \mathrm{Pd}\left({ }^{78} \mathrm{Kr}, 4 n\right){ }^{178} \mathrm{~Pb}$, at the Accelerator Laboratory of the University of Jyväskylä, Finland. A heavyion beam of krypton ${ }^{78} \mathrm{Kr}^{15+}$ was produced using an electron cyclotron resonance ion source [9] and accelerated by the $\mathrm{K}-130$ cyclotron to an energy of $358 \mathrm{MeV}$. The beam impinged on a self-supporting ${ }^{104} \mathrm{Pd}$ target with a thickness and enrichment of $745 \mu \mathrm{g} \mathrm{cm}^{-2}$ and $95.25 \%$, respectively, which 
was rotated throughout the experiment. A $34 \mu \mathrm{g} / \mathrm{cm}^{2}$ thick carbon reset foil was positioned behind the target. The beam intensity during $224 \mathrm{~h}$ of irradiation was on average $140 \mathrm{pnA}$. The recoiling nuclei of interest (recoils) were separated from the beam and unwanted reaction products using the gas-filled separator RITU [10] and transported to its focal plane, where the GREAT spectrometer [11] is located.

In the GREAT spectrometer recoils passed through a multiwire proportional counter (MWPC), where the information of energy loss, time, and position were registered. The recoils were then implanted into a set of two 300- $\mu$ $m$ thick double-sided silicon strip detectors (DSSD). Both DSSDs have an active area of $60 \times 40 \mathrm{~mm}^{2}$ and a strip pitch of $1 \mathrm{~mm}$ on both faces, yielding 4800 pixels. The amplifier gain of both horizontal and vertical strips of the DSSDs were set to measure $\alpha$-particle energies and calibrated internally using the known $\alpha$-particle energies of ${ }^{176,179} \mathrm{Hg}$ and ${ }^{176} \mathrm{Pt}$, from Refs. [12-14], respectively. A $700 \mu \mathrm{g} / \mathrm{cm}^{2}$ degrader of aluminized mylar was positioned between the DSSDs and MWPC to reduce scattered beamlike products in the implantation detectors. The time of flight (TOF) between the MWPC and DSSDs was also recorded. Two-dimensional plots of the TOF versus the energy loss signal in the MWPC and the implantation energy in the DSSDs were used to distinguish between the recoiling nuclei and beam- and targetlike particle events. An array of 14 PIN diodes with an active area of $28 \times 56 \mathrm{~mm}^{2}$ and $1 \mathrm{~mm}$ thickness each surrounds the DSSDs in the upstream position with respect to the beam axis and were used to measure escaping $\alpha$ particles. A 15 -mm-thick planar double-sided germanium strip detector with a $120 \times$ $60 \mathrm{~mm}^{2}$ active area and strip pitch of $5 \mathrm{~mm}$ was positioned directly behind the DSSDs inside the vacuum chamber. In this experiment it was used to veto energetic light-particle events that pass through the DSSDs, depositing an amount of energy that overlaps with the $\alpha$-particle energies from the nuclei of interest.

The data acquisition in the experiment was performed using the triggerless total data readout (TDR) system [15]. All events were read out individually, time stamped with a precision of $10 \mathrm{~ns}$ by a $100-\mathrm{MHz}$ metronome, and merged into a single stream of time-stamped events. The data analysis was performed using the GRAIN software package [16].

\section{RESULT AND DISCUSSION}

A two-dimensional plot of $\alpha$-particle energy parent-child correlations is illustrated in Fig. 1(a). Maximum correlation search times of 5 and $50 \mathrm{~ms}$ were used for the parent and child events, respectively. Three counts of ${ }^{178} \mathrm{~Pb}$ can be identified correlated with its child, ${ }^{174} \mathrm{Hg}$ [17]. Another event correlated with the grandchild of ${ }^{178} \mathrm{~Pb},{ }^{170} \mathrm{Pt}$ [18], can also be seen. Parent-child correlations of ${ }^{179} \mathrm{Tl}{ }^{m}$ [12], ${ }^{176} \mathrm{Hg}[12],{ }^{175} \mathrm{Hg}$ [19], and ${ }^{179} \mathrm{~Pb}[20]$ are also clearly visible. Figure $1(\mathrm{~b})$ shows the spectrum of $\alpha$-particle energies between energies of 6600 and $7800 \mathrm{keV}$ where the $\alpha$ decay of ${ }^{178} \mathrm{~Pb}$ can be seen. The shaded spectrum (blue color) represents random correlations, which were produced in the software analysis by shifting the horizontal and vertical strip numbers of the recoil implant by two. This in effect shifts the pixel with which to correlate
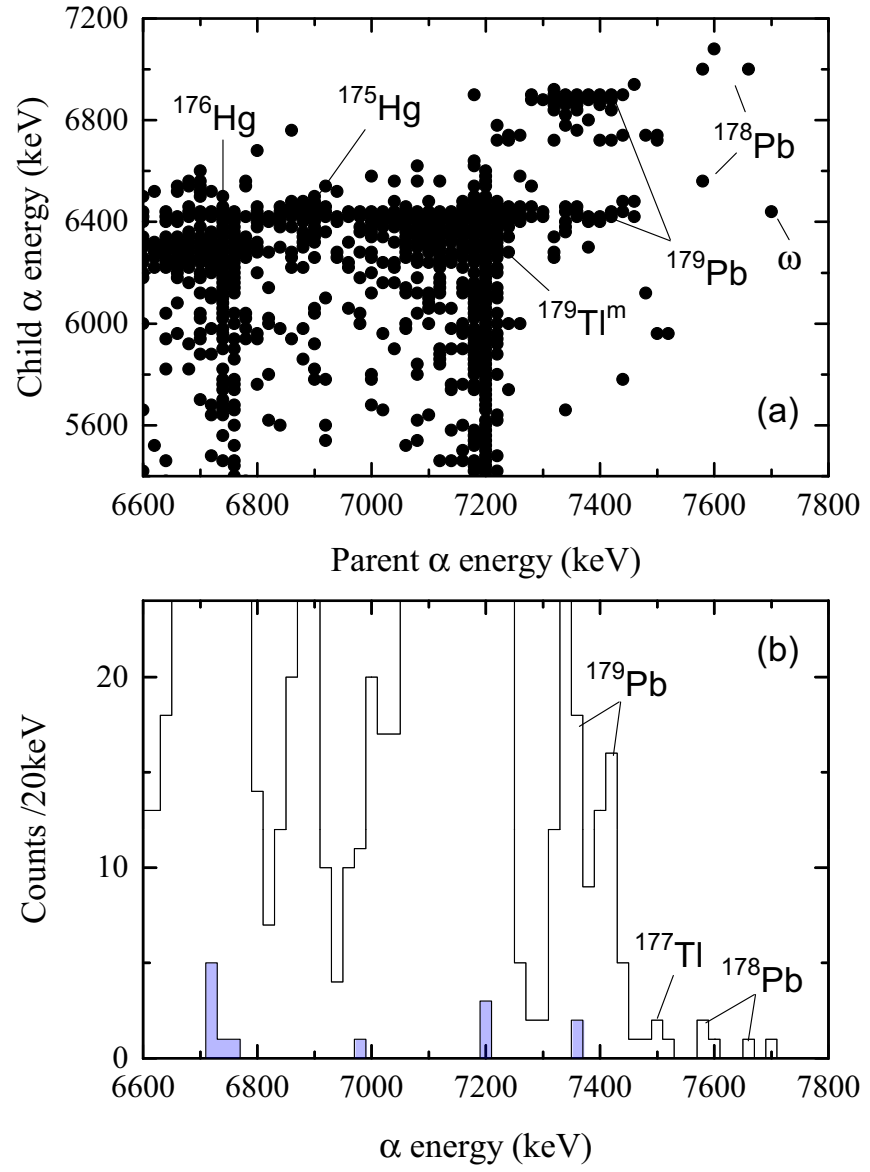

FIG. 1. (a) A matrix of parent and child $\alpha$-particle energies. Maximum searching times of 5 and $50 \mathrm{~ms}$ were used for recoil-parent pairs and parent-child pairs, respectively. The letter $\omega$ represents an unidentified $\alpha$ particle with an energy and half-life of $E_{\alpha}=7700 \mathrm{keV}$ and $t_{1 / 2} \simeq 4 \mathrm{~ms}$; (b) overlay of the $\alpha$-decay spectra within a 5 -ms correlation time, where the black line shows the real correlation and the shaded spectrum (blue color) represents the random correlations (see text for details).

the $\alpha$ decay with the recoil by two, and thus only random correlations are observed. The vanishing of counts attributed to the $\alpha$ decay of ${ }^{178} \mathrm{~Pb}$ is clear and only very few random events were observed.

In total, four counts of the new isotope ${ }^{178} \mathrm{~Pb}$ were observed. The production cross section of ${ }^{178} \mathrm{~Pb}$ is estimated to be $\sigma \simeq 4$ $\mathrm{p}$ barn, taking the calculated RITU transmission efficiency [21], DSSD efficiency, and $\alpha$-particle detection efficiency to be $50 \%, 70 \%$, and $55 \%$, respectively. It should be noted that the beam energy chosen for this reaction was optimized for a threerather than four-particle exit channel; hence the extremely low production cross section.

In order to identify all the four counts that have been assigned to ${ }^{178} \mathrm{~Pb}$, an event-by-event search along the $\alpha$-decay chain was performed. Maximum searching times of 5 and $50 \mathrm{~ms}$ were used for recoil-parent (recoil $\rightarrow{ }^{178} \mathrm{~Pb}$ ) and parent-child $\left({ }^{178} \mathrm{~Pb} \rightarrow{ }^{174} \mathrm{Hg}\right.$ ) correlations, respectively, while a 30-s searching time was applied to the rest of the decay chain, 


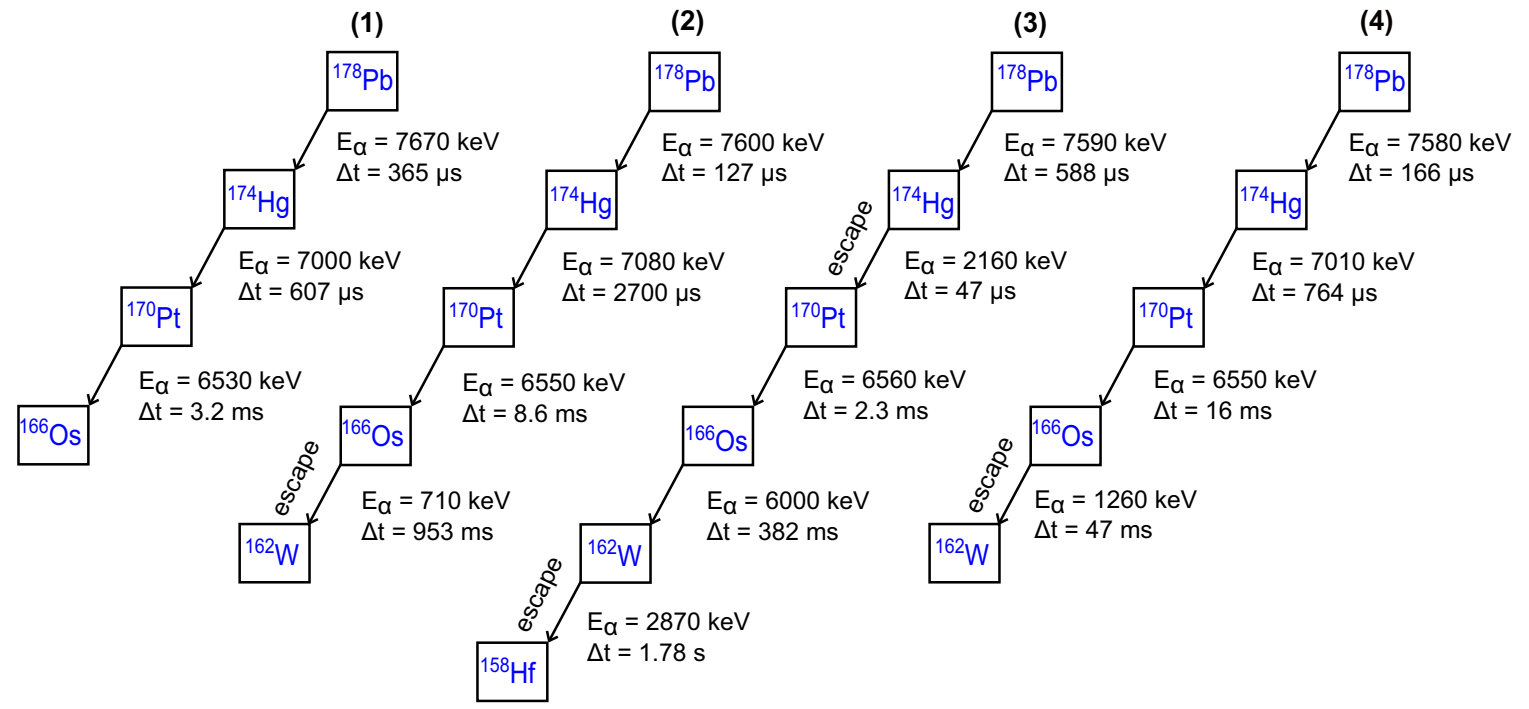

FIG. 2. $\alpha$-decay chains of ${ }^{178} \mathrm{~Pb}$. The word escape above the arrows corresponds to only part of the $\alpha$-particle energy being deposited in the DSSD, while $E_{\alpha}$ and $\Delta t$ represent the $\alpha$-particle energy detected only in the DSSD and the time difference between each generation in the chains, respectively.

which is shown below:

$$
\begin{aligned}
& { }^{178} \mathrm{~Pb} \underset{\text { chain }}{\stackrel{\alpha-\text { decay }}{\longrightarrow}} \\
& { }^{170} \mathrm{Pt} \quad[18] \underset{14(2) \mathrm{ms}}{\stackrel{6549(2) \mathrm{keV}}{\longrightarrow}} \\
& { }^{174} \mathrm{Hg} \quad[17] \underset{1.9_{-0.3}^{+0.4} \mathrm{~ms}}{\stackrel{7066(8) \mathrm{keV}}{\longrightarrow}} \\
& { }^{166} \mathrm{Os} \\
& \text { [22] } \underset{220(7) \mathrm{ms}}{\stackrel{6000(6) \mathrm{keV}}{\longrightarrow}} \\
& { }^{162} \mathrm{~W} \quad[23] \underset{0.99(3) \mathrm{s}}{\stackrel{5541(5) \mathrm{keV}}{\longrightarrow}} \\
& { }^{158} \mathrm{Hf} \\
& { }^{154} \mathrm{Yb} \quad[24] \underset{409(2) \mathrm{ms}}{\stackrel{5331(4) \mathrm{keV}}{\longrightarrow}}{ }^{150} \mathrm{Er}
\end{aligned}
$$

The four observed $\alpha$-decay chains of ${ }^{178} \mathrm{~Pb}$ are shown in Fig. 2. Chain 1 consists of three generations of full-energy $\alpha$ decays. Chains 2 and 4 show two escaping $\alpha$ particles of ${ }^{166} \mathrm{Os}$ correlated with a full energy ${ }^{178} \mathrm{~Pb} \alpha$ decay. In chain 3 two escaping $\alpha$ particles of ${ }^{174} \mathrm{Hg}$ and ${ }^{162} \mathrm{~W}$ were correlated with a full energy ${ }^{178} \mathrm{~Pb} \alpha$ decay. When an escaping $\alpha$ particle was observed in the DSSDs, the remaining energy was deposited in one of the PIN box detectors for chains 2, 3, and 4. These escaping $\alpha$ particles occur due to the shallow implantation depth of the recoil in the DSSDs, especially after the degrader of aluminized mylar was installed between the DSSDs and MWPC. Figure 2 shows that both ${ }^{178} \mathrm{~Pb} \alpha$-decay chains 2 and 4 terminate at ${ }^{162} \mathrm{~W}$. In addition, an $\alpha$ particle of ${ }^{178} \mathrm{~Pb}$ correlated beyond the $\alpha$ decay of ${ }^{166}$ Os was not observed in chain 1 , while chain 3 ends at ${ }^{158} \mathrm{Hf}$; hence the ${ }^{158} \mathrm{Hf} \rightarrow{ }^{154} \mathrm{Yb}$ $\alpha$ decay was not observed. These end points are caused by the $\alpha$-branching ratios of ${ }^{166} \mathrm{Os},{ }^{162} \mathrm{~W}$, and ${ }^{158} \mathrm{Hf}$ having values of $72(13) \%, 44(3) \%$, and 45(2)\% [24], respectively.

The half-life of the ground state of ${ }^{178} \mathrm{~Pb}$ was determined to be $t_{1 / 2}=0.21_{-0.08}^{+0.21} \mathrm{~ms}$ using the maximum-likelihood method. In Fig. 3 the logarithmic-time difference spectrum between the implanted recoils in the DSSDs and the $\alpha$ decay of ${ }^{178} \mathrm{~Pb}$ is plotted. The decay constant value obtained from the maximumlikelihood method as stated previously was used to produce the line curve (shown in red color online) plotted in Fig. 3, where the maximum value of the distribution, as described in Ref. [25], yields $\ln (1 / \lambda)$, where $\lambda$ is the decay constant. The standard deviation value of $\sigma_{\exp }=0.58$ was obtained from the distribution, which is within one $\sigma$ of the calculated expectation value of $\sigma_{\text {calc }}=0.98$ and falls well between the lower and upper limits of $0.31 \leqslant \sigma_{\text {calc }} \leqslant 1.92$ calculated in Ref. [25], thus confirming that the four events assigned here to ${ }^{178} \mathrm{~Pb}$ originate from the same radioactive decay.

The experimental $\alpha$-decay spectroscopic factor $S^{\exp }$ was calculated for even- $A$ lead nuclei between $96 \leqslant N \leqslant 112$ including the experimental $\alpha$-decay half-life of ${ }^{178} \mathrm{~Pb}$ from this work, using the formula, $S^{\exp }=t_{1 / 2}^{\text {calc }} / t_{1 / 2}^{\exp }$, where $t_{1 / 2}^{\exp }$ is

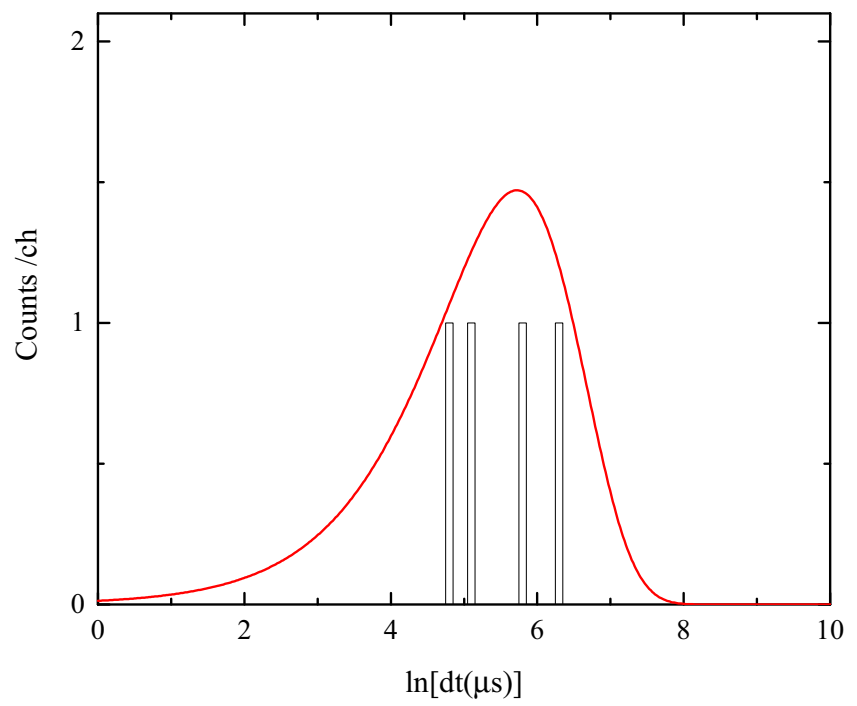

FIG. 3. Time spectrum of the ${ }^{178} \mathrm{~Pb} \alpha$ decay, which shows the logarithm of the time difference $\ln (d t)$ between the implanted recoil and $\alpha$ decay of ${ }^{178} \mathrm{~Pb}$ within a $5 \mathrm{~ms}$ correlation-searching time. The red line is a plotted distribution described in Ref. [25] using decay constant value from the maximum-likelihood method. 
the experimental $\alpha$-decay half-life. The theoretical $\alpha$-decay half-life $t_{1 / 2}^{\text {calc }}$ values were taken from Ref. [8] using two different models, the Coulomb and proximity potential model (CPPM) and the analytical formulas of Royer et al. [26] using the generalized liquid drop model (GLDM) and the semi-empirical Rasmussen method [27] was also used for comparison.

Figure 4(a) shows a comparison of the calculated experimental $\alpha$-decay spectroscopic factor $S^{\exp }$ using the three different models as a function of neutron number. The experimental half-life value of ${ }^{178} \mathrm{~Pb}$ is consistent within errors with the theoretical value calculated using the CPPM as can be noted in Fig. 4(a), where the spectroscopic factor $S \simeq 1$. The plot reveals an overestimation of the theoretical half-life using the GLDM model up to $N=106$ where a shift to an underestimation is observed up to $N=112$. The reverse trend is observed for the CPPM. Generally, there is a good agreement between the experimental values and the theoretical calculations, especially for the Rassmussen method [27]. However, the $S^{\exp }$ values for $N=108$ and $N=110$ using the CPPM show a disagreement. As the agreement with the GLDM for these neutron numbers remains good, this points toward a failure in CPPM to reproduce an accurate partial half-life for these mass numbers.

The average energy of $E_{\alpha}=7610(30) \mathrm{keV}$ was deduced for the $\alpha$ decay of ${ }^{178} \mathrm{~Pb}$. This value is consistent within the error with the energy value in Ref. [1]. The error in the average energy was calculated as the sum of the mean and the systematic error (deviations between calibrated and reference energies).

The $\alpha$-decay hindrance factor was calculated and it corresponds to an unhindered (favored) transition with $\mathrm{HF}=$ $t_{1 / 2}^{\exp } / t_{1 / 2}^{r a s}=0.8$, where $t_{1 / 2}^{\exp }$ is the experimental half-life from this work and $t_{1 / 2}^{\text {ras }}$ is the theoretical $\alpha$-decay half-life calculated using the Rasmussen method [27].

The $\alpha$-decay reduced width, $\delta_{\alpha}^{2}=\lambda_{\exp } \times h / p=$ $92_{-31}^{+92} \mathrm{keV}$, for the $\alpha$-decay energy of $E_{\alpha}=7610$ (30) $\mathrm{keV}$ was deduced for this decay with an assumption of $\Delta l=0$, where $h$ is Planck constant and $p$ is the barrier penetration factor calculated using the WKB approximation. The calculated reduced width value of ${ }^{178} \mathrm{~Pb}_{96}$ is consistent with the $N=96$ isotones ${ }^{176} \mathrm{Hg}_{96}$ and ${ }^{174} \mathrm{Pt}_{96}$ having values of $\delta_{\alpha}^{2}=$ $85(13) \mathrm{keV}$ and $\delta_{\alpha}^{2}=95(2) \mathrm{keV}$, respectively. The reduced $\alpha$-decay width calculation for ${ }^{176} \mathrm{Hg}$ and ${ }^{174} \mathrm{Pt}$ were also performed using the Rasmussen method [27] and include the experimental values taken from Refs. [33] and [24], respectively. The above calculations assume that the $\alpha$ decay of ${ }^{178} \mathrm{~Pb}$ has a branching ratio of $100 \%$. This assumption is based on the fact that the half-life predictions from Möller et al. [36] for the $\alpha$ and $\beta$ decay of ${ }^{178} \mathrm{~Pb}$ are $t_{1 / 2}^{\alpha}=5.012 \times 10^{-5} \mathrm{~s}$ and $t_{1 / 2}^{\beta}=$ $0.2815 \mathrm{~s}$, respectively, which yield a theoretical branching ratio of $b_{\alpha}=99.98 \%$. Therefore, the large error in the ${ }^{178} \mathrm{~Pb}$ half-life causes the large uncertainty in the $\alpha$-decay reduced-width value, while the accuracy of the ${ }^{178} \mathrm{~Pb}$ $\alpha$-particle energy is not a major factor.

Figure 4(b) provides the $\alpha$-decay reduced width for even $\mathrm{Pb}, \mathrm{Hg}$, and $\mathrm{Pt}$ nuclei including the ${ }^{178} \mathrm{~Pb}$ value from this work

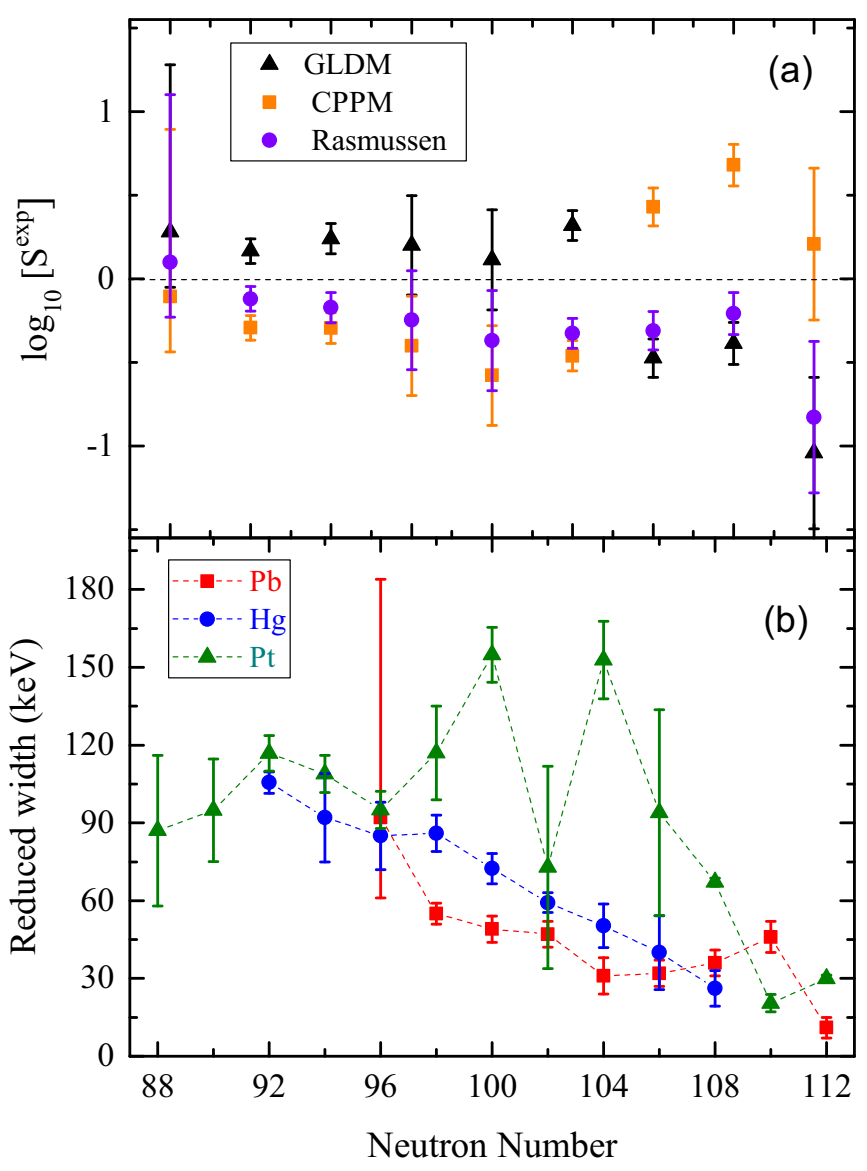

FIG. 4. (a) Logarithmic experimental spectroscopic-factor log ${ }_{10}\left[S^{\exp }\right]$ for even- $A$ lead isotopes. The theoretical $\alpha$-decay half-life values were obtained from Refs. [8,27] using the CCPM, the GLDM models, and Rasmussen method, respectively; (b) $\alpha$-decay reducedwidth values as function of neutron number for even- $A$ lead, mercury, and platinum nuclei. The experimental values of the $\mathrm{Pb}$ isotopes in both panels (a) and (b) are obtained from Refs. [5,28-34], while the experimental values used in $\mathrm{Hg}$ and $\mathrm{Pt}$ reduced-width calculation were obtained from ENSDF [35].

as a function of neutron number between $88 \leqslant N \leqslant 112$. The plot shows that the reduced widths of lightest lead isotopes are greater than those of the midshell region and the new value for ${ }^{178} \mathrm{~Pb}$ continues this trend. The same general trend is also followed by the $\mathrm{Hg}$ isotopes, while the Pt nuclei are markedly different. A key point in the Pt systematics is the downturn in the reduced widths below $N=92$. While there is an overall trend of increasing magnitude as the neutron number decreases, some other features are apparent in the $\mathrm{Pb}$ isotopic chain. There is an increase below $N=112$ and then again at $N=98$, which coincide with the closing and opening of the $i_{13 / 2}$ neutron orbital. The large deviations around the neutron midshell, especially for the Pt nuclei, are likely due to inaccurate branching ratios. It can be speculated that the increase at $N=96$, when approaching the proton drip line for

${ }^{178} \mathrm{~Pb}$, is a consequence of the two-proton separation energy becoming negative, $S_{2 p}^{\exp }=-798(44) \mathrm{keV}\left(S_{2 p}^{\text {calc }}=-470 \mathrm{keV}\right.$ [36]), while for ${ }^{180} \mathrm{~Pb}$ at $N=98$ the protons are still bound 


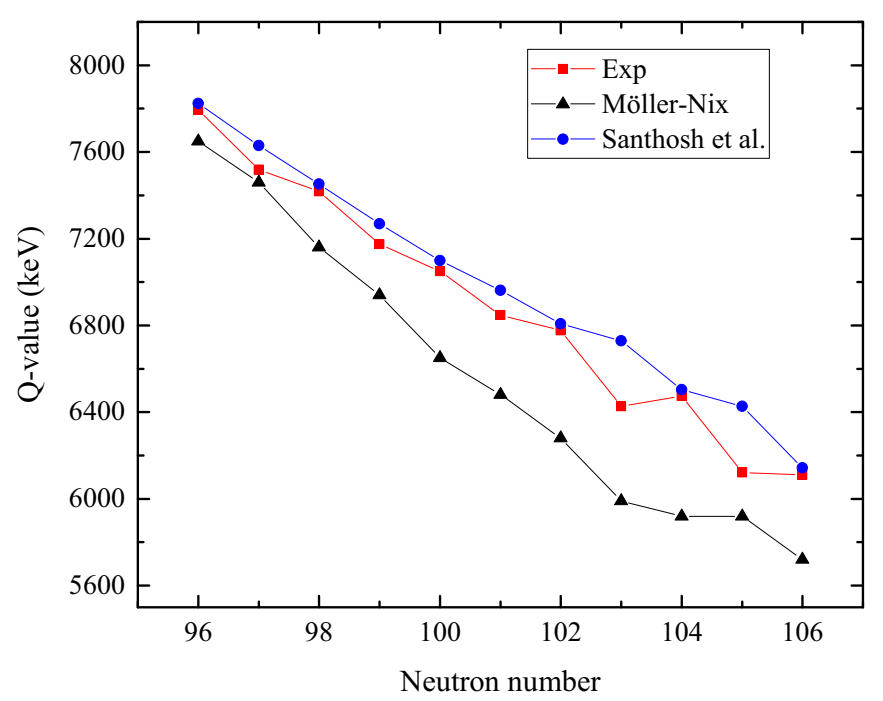

FIG. 5. The $\alpha$-decay $Q$ values as a function of the neutron number for different lead isotopes. The filled triangles represent the experimental values including the ${ }^{178} \mathrm{~Pb} Q$ value from this work. The filled squares and circles show the values obtained from Möller et al. [36] and Santhosh et al. [8], respectively.

with $S_{2 p}^{\text {exp }}=191(18) \mathrm{keV}\left(S_{2 p}^{\text {calc }}=590 \mathrm{keV}\right.$ [36]). Of course, two neutrons are also required to form an $\alpha$ particle. The two-neutron separation energy for ${ }^{178} \mathrm{~Pb}$ is $S_{2 n}=22.13 \mathrm{MeV}$ and this value increases as the $N=82$ shell closure is approached beyond the proton drip line. Still, this weak binding of the protons may further enhance the probability of $\alpha$-particle preformation and subsequent decay. Contrary to this the Pt chain of reduced widths starts a downward trend as the nuclei become 2 proton unbound, yet the $\mathrm{Hg}$ nuclei continue the upward trend after 2-proton separation energy becomes negative. Clearly, more precise data on both half-lives and branching ratios for many isotopes are still needed to have a clear picture of the systematic behavior of reduced $\alpha$ widths in vicinity of $Z=82$.
The $Q$ value of $Q_{\alpha}=7785(24) \mathrm{keV}$ was calculated for the ${ }^{178} \mathrm{~Pb} \rightarrow{ }^{174} \mathrm{Hg} \alpha$ decay. The mass excess of $3603(42) \mathrm{keV}$ was deduced as well using a ${ }^{174} \mathrm{Hg}$ mass excess value of 6607(34) keV from Ref. [17]. This value is consistent within the error with the value of 3608(39) $\mathrm{keV}$ reported in Ref. [1] and found to be comparable to the predicted value of $3430 \mathrm{keV}$ for ${ }^{178} \mathrm{~Pb}$ by Möller et al. [37].

Figure 5 compares the experimental $\alpha$-decay $Q$ values for lead isotopes between $178 \leqslant A \leqslant 188$ with those obtained by Möller et al. [36] using microscopic-macroscopic calculations and values obtained from Santhosh et al. [8]. The $Q$-value calculation in Ref. [8] was achieved by using the mass-excess values of the parent, child nuclei, and the $\alpha$ particle from Ref. [38] plus the atomic-electron screening effect term from Ref. [39]. The graph reveals that there is a good agreement between the experimental values including the ${ }^{178} \mathrm{~Pb}$ value from this work and those calculated in Ref. [8].

\section{CONCLUSION}

In conclusion, this paper reports the confirmation of the new isotope ${ }^{178} \mathrm{~Pb}$. The $\alpha$-particle energy and $Q$ value of $E_{\alpha}=7610(30) \mathrm{keV}$ and $Q_{\alpha}=7785(24) \mathrm{keV}$, respectively, were measured. Also, a half-life of $t_{1 / 2}=0.21_{-0.08}^{+0.21} \mathrm{~ms}$ was measured for ${ }^{178} \mathrm{~Pb}$ and is in agreement with the CPPM calculations by Santhosh et al. [8]. The mass excess value of $3603(42) \mathrm{keV}$ is in good agreement with the one deduced by Batchelder et al. [1]. The $\alpha$-decay reduced width indicates a $\Delta l=0$ character for the observed transition.

\section{ACKNOWLEDGMENTS}

The authors would like to thank John Green from Argonne National Laboratory and B. Lommel and the GSI target laboratory staff for producing the Pd targets. This work has been supported by the Academy of Finland under the Finnish Center of Excellence Program (Contract No. 213503), the Marie Curie Career Integration Grant (Grant No. 304033), and the Academy of Finland (Grant No. 257562).
[1] J. C. Batchelder, K. S. Toth, M. W. Rowe, T. N. Ginter, K. E. Gregorich, V. E. Ninov, F. Q. Guo, J. Powell, X.-J. Xu, and J. Cerny, AIP Conf. Proc. 681, 144 (2003).

[2] A. Gottardo, J. J. Valiente-Dobón, G. Benzoni, R. Nicolini, A. Gadea, S. Lunardi, P. Boutachkov, A. M. Bruce, M. Górska, J. Grebosz et al., Phys. Rev. Lett. 109, 162502 (2012).

[3] P. Van Duppen and M. Huyse, Hyperfine Interact. 129, 149 (2000).

[4] J. Wauters, N. Bijnens, P. Dendooven, M. Huyse, H. Y. Hwang, G. Reusen, J. von Schwarzenberg, P. Van Duppen, R. Kirchner, and E. Roeckl (ISOLDE Collaboration), Phys. Rev. Lett. 72, 1329 (1994).

[5] A. N. Andreyev, N. Bijnens, M. Huyse, P. V. Duppen, M. Leino, T. Enqvist, P. Kuusiniemi, W. Trzaska, J. Uusitalo, N. Fotiades et al., J. Phys. G: Nucl. Part. Phys. 25, 835 (1999).

[6] A. N. Andreyev, M. Huyse, P. Van Duppen, C. Qi, R. J. Liotta, S. Antalic, D. Ackermann, S. Franchoo, F. P. Heßberger, S. Hofmann et al., Phys. Rev. Lett. 110, 242502 (2013).
[7] C. Qi, A. Andreyev, M. Huyse, R. Liotta, P. V. Duppen, and R. Wyss, Phys. Lett. B 734, 203 (2014).

[8] K. P. Santhosh, I. Sukumaran, and B. Priyanka, Nucl. Phys. A 935, 28 (2015).

[9] H. Koivisto, P. Heikkinen, V. Hänninen, A. Lassila, H. Leinonen, V. Nieminen, J. Pakarinen, K. Ranttila, J. Arje, and E. Liukkonen, Nucl. Instrum. Methods Phys. Res., Sect. B 174, 379 (2001).

[10] M. Leino, J. Äystö, T. Enqvist, P. Heikkinen, A. Jokinen, M. Nurmia, A. Ostrowski, W. H. Trzaska, J. Uusitalo, K. Eskola et al., Nucl. Instrum. Methods Phys. Res. B99, 653 (1995).

[11] R. D. Page, A. N. Andreyev, D. E. Appelbe, P. A. Butler, S. J. Freeman, P. T. Greenlees, R. D. Herzberg, D. G. Jenkins, G. D. Jones, P. Jones et al., Nucl. Instrum. Methods Phys. Res. B204, 634 (2003).

[12] A. N. Andreyev, V. Liberati, S. Antalic, D. Ackermann, A. Barzakh, N. Bree, T. E. Cocolios, J. Diriken, J. Elseviers, D. Fedorov et al., Phys. Rev. C 87, 054311 (2013).

[13] C. M. Baglin, Nucl. Data Sheets 110, 265 (2009). 
[14] B. Singh, Nucl. Data Sheets 75, 199 (1995).

[15] I. H. Lazarus, D. E. Appelbe, P. A. Butler, P. J. Coleman-Smith, J. R. Cresswell, S. J. Freeman, R. D. Herzberg, I. Hibbert, D. T. Joss, S. C. Letts et al., IEEE Trans. Nucl. Sci. 48, 567 (2001).

[16] P. Rahkila, Nucl. Instrum. Methods Phys. Res., Sect. A A595, 637 (2008).

[17] D. Seweryniak, J. Uusitalo, M. P. Carpenter, D. Nisius, C. N. Davids, C. R. Bingham, L. T. Brown, L. Conticchio, D. J. Henderson, R. V. F. Janssens et al., Phys. Rev. C 60, 031304 (1999).

[18] H. Kettunen, T. Enqvist, T. Grahn, P. T. Greenlees, P. Jones, R. Julin, S. Juutinen, A. Keenan, P. Kuusiniemi, M. Leino et al., Phys. Rev. C 69, 054323 (2004).

[19] D. O’Donnell, J. Simpson, C. Scholey, T. Bäck, P. T. Greenlees, U. Jakobsson, P. Jones, D. T. Joss, D. S. Judson, R. Julin et al., Phys. Rev. C 79, 051304 (2009).

[20] H. Badran et al. (unpublished).

[21] J. Sarén, J. Uusitalo, M. Leino, and J. Sorri, Nucl. Instrum. Methods Phys. Res., Sect. A 654, 508 (2011).

[22] D. E. Appelbe, J. Simpson, M. Muikku, H. J. Boardman, A. Melarangi, R. D. Page, P. T. Greenlees, P. M. Jones, R. Julin, S. Juutinen et al., Phys. Rev. C 66, 014309 (2002).

[23] H. J. Li, B. Cederwall, T. Back, C. Qi, M. Doncel, U. Jakobsson, K. Auranen, S. Bonig, M. C. Drummond, T. Grahn et al., Phys. Rev. C 92, 014326 (2015).

[24] R. D. Page, P. J. Woods, R. A. Cunningham, T. Davinson, N. J. Davis, A. N. James, K. Livingston, P. J. Sellin, and A. C. Shotter, Phys. Rev. C 53, 660 (1996).

[25] K. H. Schmidt, Eur. Phys. J. A 8, 141 (2000).
[26] G. Royer, J. Phys. G 26, 1149 (2000).

[27] J. O. Rasmussen, Phys. Rev. 113, 1593 (1959).

[28] P. Rahkila, D. G. Jenkins, J. Pakarinen, C. Gray-Jones, P. T. Greenlees, U. Jakobsson, P. Jones, R. Julin, S. Juutinen, S. Ketelhut et al., Phys. Rev. C 82, 011303 (2010).

[29] M. Venhart et al., Eur. Phys. J. A 48, 101 (2012).

[30] A. N. Andreyev, D. Ackermann, F. P. Hessberger, K. Heyde, S. Hofmann, M. Huyse, D. Karlgren, I. Kojouharov, B. Kindler, B. Lommel et al., Phys. Rev. C 69, 054308 (2004).

[31] N. Bijnens, I. Ahmad, A. N. Andreyev, J. C. Batchelder, C. R. Bingham, D. Blumenthal, B. C. Busse, X. S. Chen, L. F. Conticchio, C. N. Davids et al., Z. Phys. A 356, 3 (1996).

[32] J. D. Richards, C. R. Bingham, Y. A. Akovali, J. A. Becker, E. A. Henry, P. Joshi, J. Kormicki, P. F. Mantica, K. S. Toth, J. Wauters et al., Phys. Rev. C 54, 2041 (1996).

[33] K. S. Toth, C. R. Bingham, J. C. Batchelder, L. T. Brown, L. F. Conticchio, C. N. Davids, R. J. Irvine, D. Seweryniak, D. M. Moltz, W. B. Walters et al., Phys. Rev. C 60, 011302 (1999).

[34] Y. Akovali, Nucl. Data Sheets 84, 1 (1998).

[35] Evaluated Nuclear Structure Data Files (ensdf) [http://www.nndc.bnl.gov/ensdf/].

[36] P. Möller, J. R. Nix, and K. L. Kratz, At. Data Nucl. Data Tables 66, 131 (1997).

[37] P. Möller, J. Nix, W. Myers, and W. Swiatecki, At. Data Nucl. Data Tables 59, 185 (1995).

[38] M. Wang, G. Audi, A. H. Wapstra, F. G. Kondev, M. MacCormick, X. Xu, and B. Pfeiffer, Chin. Phys. C 36, 1603 (2012).

[39] K.-N. Huang, M. Aoyagi, M. H. Chen, B. Crasemann, and H. Mark, At. Data Nucl. Data Tables 18, 243 (1976). 